\title{
Communication: the good, the bad, and the painful
}

\author{
Navjoyt Ladher clinical editor, The BMJ
}

Thoughtful and effective communication in medicine, as in life, can be difficult. Even everyday encounters with patients can present opportunities to reflect, learn, and adapt.

I have always thought that warning patients about the pain they may feel during a procedure was an important part of informed consent, but I realise that despite my best intentions for communication that is good and patient centred, there may be potential for harm. In a personal view Baruch Krauss explains how expectations are partly responsible for shaping the response to a stimulus (doi:10.1136/bmj.h649). Krauss encourages practitioners to adopt language that is less leading when explaining procedures to patients. Telling patients "this may hurt" can lead to a "nocebo" effect, where negative expectations might enhance a negative response. On the other hand "you may feel something now" allows for a spectrum of responses.

In his latest Ethics Man column Daniel Sokol describes the conflicts that can arise when balancing cultural sensitivity with ethical and legal duties (doi:10.1136/bmj.h850). Doctors treating patients from cultures that differ greatly from their own may fall into difficulty, illustrated by the obstetric trainee who faced the first UK prosecution for female genital mutilation (doi:10. 1136/bmj.h703). He was acquitted, with the jury needing less than half an hour of deliberation to find him not guilty. The Crown Prosecution Service's decision to press charges was an unwise one, says Margaret McCartney (doi:10.1136/bmj.h815), particularly when it has yet to prosecute anyone for taking girls abroad from the UK to undergo genital mutilation.
Our Head to Head this week asks whether patients can use test results effectively if they have direct access (doi:10.1136/bmj. h673). Brian Zikmund-Fisher thinks we need to get creative with how we communicate results if patients are to be able to use them meaningfully. He suggests using visual cues to better frame test results so that patients can interpret results and place them in context and use them to guide self monitoring or inform decision making. Arguing the other side, Maurice O'Kane and Danielle Freedman point to the high levels of satisfaction among patients who have had direct access to test results.

Our new What Your Patient Is Thinking series of Education articles debuted last month to an overwhelming response (doi:10. 1136/bmj.g6845). In the second article of the series Sarah Chapman describes the challenges of medical consultations for people with hearing loss (doi:10.1136/bmj.h184) and encourages readers to ask the empowering six word question, "How can I help you hear?" As Chapman describes, "It's a respectful, empathetic, and practical opening question, inviting specific instructions that you can follow, knowing that you are doing the right things to enable a helpful dialogue. I'm immediately made to feel that you're on my side, that we're partners in this business of managing my health, and I'm able to tell you what I need you to do."

Six words that can convey all this: that is the art of good communication. 